\title{
ETHNOLOGISCHE BEOBACHTUNGEN IN DER GUAJIRA HALBINSEL
}

Hans J. Tanner

Mit 6 Abbildungen

\section{ALLGEMEINES}

Die bedeutendste Halbinsel der südamerikanisch-karibischen Nordküste ist die zu Kolumbien und Venezuela gehörende Guajira Halbinsel. Sie ist auch heute noch fast ausschließlich von reinen Indianern bewohnt, von denen nur ein Bruchteil «zivilisiert» ist und sich zum Christentum bekennt. Diese Guajira Indianer gehören nach KRICKeberg ${ }^{1}$ zu den Aruakstämmen, die vom Hochland von Guayana ausgehend, sich allmählich über große Gebiete Südamerikas ausgebreitet hatten. Einstmals wohl fast die ganze Nordküste Südamerikas innehaltend, sind sie heute zum größten Teil wieder verschwunden. Neben ihnen als dem zahlenmäßig bedeutendsten Restvolk, sind u. a. noch Aruakindianer im Delta des Orinoco zu finden. Die Guajira haben sich nach der Eroberung Südamerikas durch die Spanier aus Ackerbauern zu einem Viehzüchtervolk gewandelt. Heute noch ist die Viehzucht- und zwar namentlich die Ziegen- und Schafzucht - ihre wichtigste Lebensgrundlage, und neben der Jagd die Hauptbeschäftigung des Großteils der Männer, während die Frauen sich neben der Arbeit im Haus, besonders der Bestellung der kärglichen Äcker widmen. Daneben aber hat sich an der Westküste der Guajira, zwischen Cabo la Vela und Rio Hacha eine allerdings wenig zahlreiche Fischerbevölkerung herangebildet, die bis vor etwa 2 Jahren neben dem Fischfang in dem relativ ruhigen Meer dem Tauchen nach Perlen oblag. Gegenwärtig ist das Perlensuchen ganz eingestellt; statt dessen tauchen die Fischer nach Langusten, die von Großhändlern aufgekauft und mit Flugzeugen zum Verbrauch nach Barranquilla und ins Landesinnere Kolumbiens versandt werden.

Eine wichtige Erwerbsquelle für die Guajira Indianer bildet sodann die Arbeit in den von der Kolumbianischen Banco del la Republica betriebenen Salinen in Manaure und das Einsammeln der für die Gerberei wichtigen Dividivifrüchte. Erwähnen wir noch das Abschälen von den ebenfalls in der Gerberei verwendeten Mangroverinden (Mangle), die besonders aus den Mangrovewäldern rund um die großen Buchten an der Nordküste der Halbinsel (Portete, Bahia Hondita) gewonnen werden und das Graben nach Gips, namentlich in den miozänen und postmiozänen Ablagerungen längs der Westküste und in der im Innern gelegenen Porchinagegend, dann haben wir die Existenzgrundlage der «nicht zivilisierten» Guajira Indianer umrissen.

Die sogenannten „Civilisados“ und namentlich viele Mestizen betätigen sich fast ausschließlich als Händler und Schankwirte. Hersteller von Rhum, als Zwischenhändler in der Ausfuhr der Landesprodukte, oder sie haben Teil am ausgiebigen, wohl ausgebauten Schmuggel, der beträchtliche Warenmengen, namentlich Zigaretten, Mehl, Stoffe und Schnäpse von den Freihäfen auf Curacao und Aruba her über die Hauptschmuggelhäfen Pto. Lopez, Pto. Estrella, Bahia Honda in die Halbinsel bringt, von wo sie nachher den Weg ins Landesinnere Kolumbiens und nach Venezuela hinüber finden. Dieser Schmuggel mit seinem regen Lastwagenverkehr (es existieren eine Reihe während der Trockenzeit befahrbarer Autopisten, die z. T. von den Schmugglern selbst aus dem Dornbusch ausgehauen wurden, der das semiaride Land weithin bedeckt), seinem Zufluß von Geld und billigem Alkohol, ist neben der Anziehungskraft der relativ nahe gelegenen Großstadt Maracaibo ein Hauptgrund für den beginnenden Zerfall der Sitten und Gebräuche der Guajira Indianer. Viele Indianer sind im Laufe der letzten Zeit, z. T. infolge einer Reihe extrem trockener Jahre, die große Lücken in die Viehherden der Guajiros schlugen und namentlich das Großvieh sehr stark dezimierten, z. T. 1939.

1 W. Krickeberg, Amerika, in: H. A. Bernatzik. Die große Völkerkunde. Band III, Leipzig 


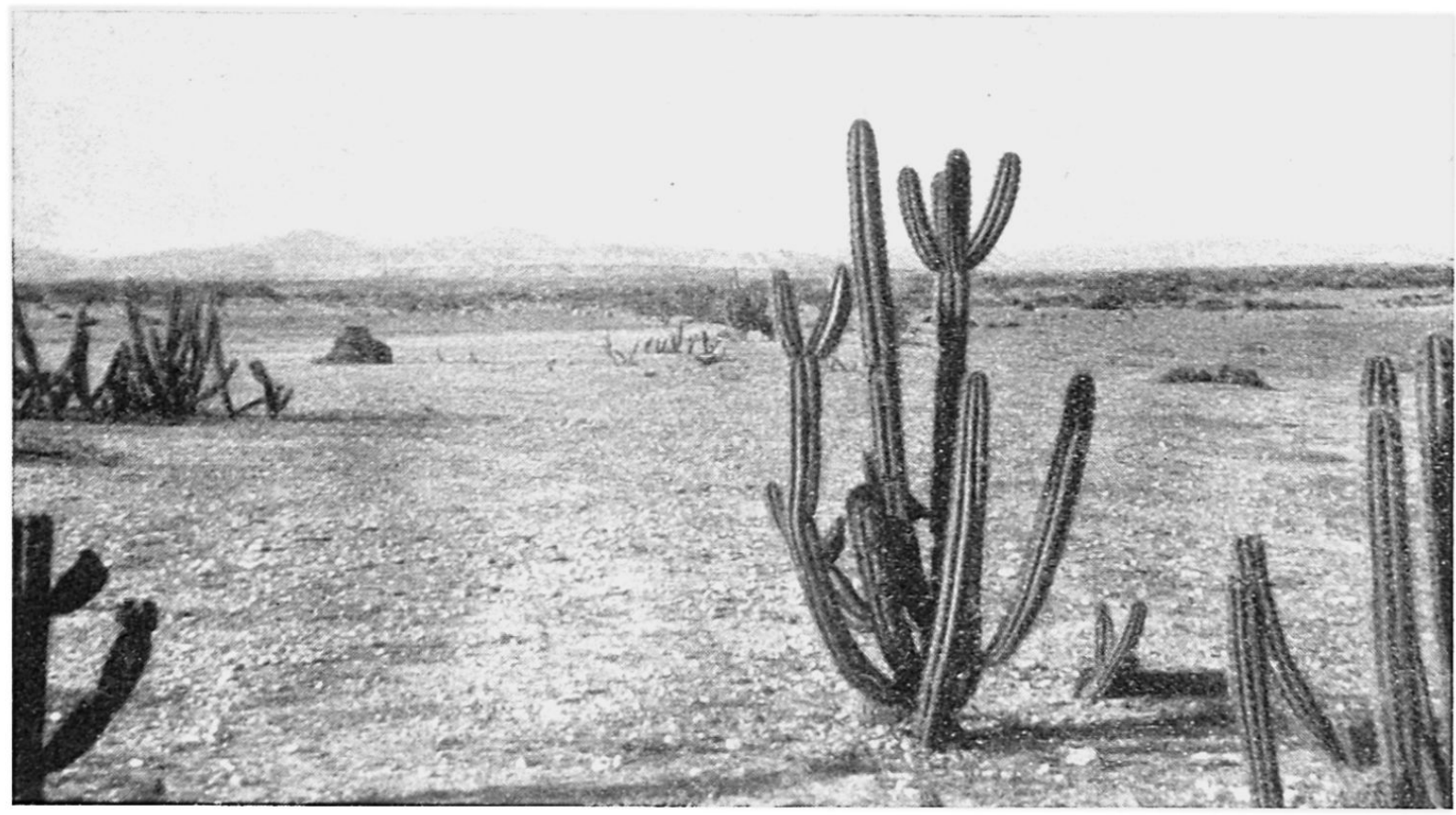

Abb. 1 Halbwüste im Innern der Guajirahalbinsel bei Porchina. In der Regenzeit überzieht sich der Boden mit magerm Gras. Im Hintergrund die Serra da Jarara.

Photo H. TANNER, 16. 4. 1950

wegen blutiger $\mathrm{F}$ amilienfehden, oder auch dank der aufmunternden Einwanderungspolitik der früheren venezolanischen Regierung General Medinas nach Venezuela ausgewandert. Man trifft heute Guajira Indianer in den Dörfern entlang der Sierra de Perija bis Machiques, der Großteil der Auswanderer blieb aber in Maracaibo selbst hängen, wo im Vorort Ziruma eine ausgedehnte Indianersiedlung entstanden ist. Die Männer betätigen sich in der Stadt als Handlanger, die Frauen aber verfallen in diesem Oelboomgebiet mit seiner Großzahl gut bezahlter Arbeiter nur allzuleicht der Prostitution. $\mathrm{Da}$ die Guajiros sich kaum dauernd außerhalb der Halbinsel niederlassen wollen, sondern immer wieder nach einer Reihe von Jahren in ihre Heimat zurückwandern, wurden Geschlechtskrankheiten in großem Stil in die Halbinsel eingeschleppt. Auch die Ersparnisse der Männer wirken sich kaum segenbringend aus, denn die Indianer wissen mit dem Geld meist nichts Rechtes anzufangen und vertrinken ihre Barschaft in kurzer Zeit. So kommt es, daß immer mehr Schankstellen aus dem Boden schießen, immer mehr Schnapsbrennereien in Betrieb gesetzt werden und immer mehr betrunkene Männer und neuerdings auch Frauen anzutreffen sind. Da die Indianer sich sehr oft im Rauschzustand bei den Schankwirten verschulden, bleibt ihnen nichts anderes übrig, als nach Maracaibo zurückzukehren, um mehr Geld zu verdienen. Nicht zuletzt deshalb hat sich im Verlaufe der letzten Jahre ein reger Busverkehr zwischen der Guajirahalbinsel und Maracaibo entwickelt (in zweitägiger Fahrt kann man in der Trockenzeit von jedem Punkt der Guajira aus Maracaibo erreichen) und ein stetes Hin- und Zurückwandern vieler Indianer setzte ein, so daß der beginnende Verfall der Sitten und Gebräuche nicht ausblieb.

In der Guajira arbeitet seit Anfang dieses Jahrhunderts eine spanische Kapuzinermission. Wenn auch die Zahl der konvertierten Indianer immer noch recht klein ist, so ist es doch wohl hauptsächlich dem Einfluß der Mission zu verdanken, daß die Guajiros ihre früher traditionelle Feindschaft gegenüber den Fremden weitgehend abgelegt haben. Während noch 1925, als STUTZER ${ }^{2}$ die Guajira bereiste, der Besuch vieler Gegenden mit ernsten Gefahren verbunden war, läuft der Reisende heute beim korrekten Verhalten keine besonderen Gefahren mehr. STUTZER schätzte 1925 die Zahl der Guajiros auf ca. 15000 Menschen, PıсноN errechnete für 1943 ca. 26000 Indianer, die 25 verschiedenen Familien angehörten (größte Familien: Epieyu und Uriana mit je ca. 4500 Menschen). ${ }^{3}$ Im März 1950 schätzte der kolumbianische Regierungskommissär die Zahl der Indianer auf 10000 , der Leiter der Missionsschule Nazareth aber auf 25000 (persönliche Mitteilung). Diese großen Unterschiede in den Zahlenangaben beruhen darauf, daß die Guajiros, obwohl keine Nomaden, einem starken Wandertrieb unterliegen und beständig große Teile der Bevölkerung nicht an ihren Wohnstätten, sondern auf der Wanderung zu Familienbesuchen, nach Wasserlöchern oder zur Arbeitsannahme in den Salinen von Manaure oder in Maracaibo zu treffen sind. Mir scheint aber die letzte Schätzung die richtige zu sein. Ich stütze mich dabei auf die Beobachtung, daß wir bei unserem Besuch in Manaure im April des Jahres 1950 über 1000 Indianer gleichzeitig bei der Arbeit in den Salinen trafen, und

2 O. STUTzer: Streifzüge eines Geologen im Gebiet der Guajira Indianer. Berlin 1927.

3 F. D. Pichon: Geografia de la Peninsula Guajira, Primera Edition, Santa Marta, Colombia, 1947. 


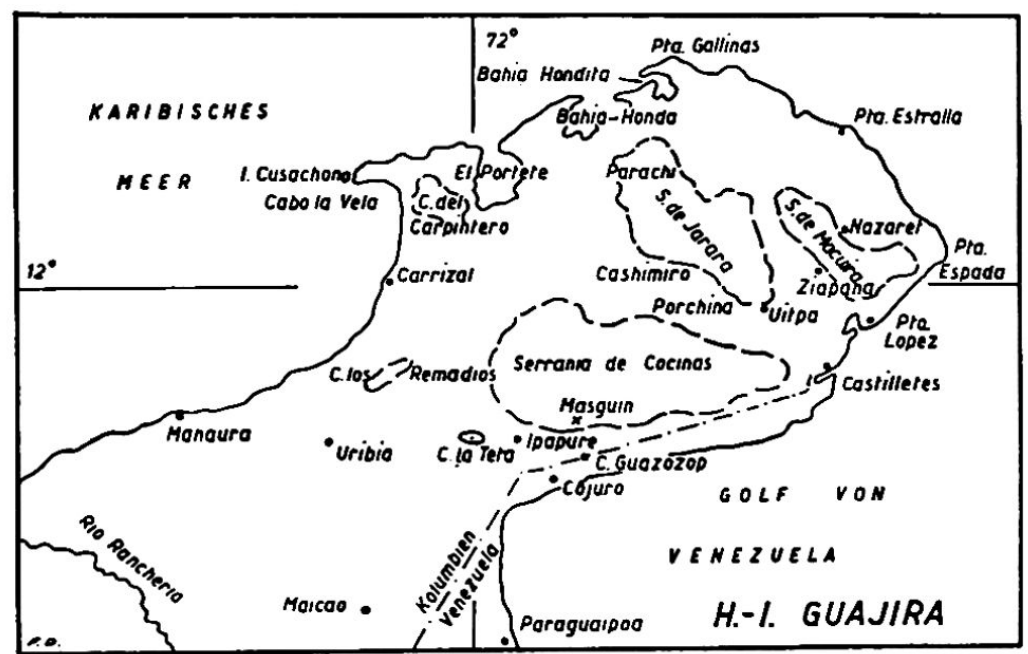

Abb. 2

Skizze des Untersuchungsgebietes mit den im Text erwähnten Namen.

daß wir verschiedenen Tanzfesten und Begräbniszeremonien beiwohnten, die, trotzdem sie sich in den weniger dicht besiedelten Gebieten der Halbinssl abspielten, oft von mehreren hundert Indianern besucht waren.

Nach Pichon ist die Flächenausdehnung der Guajira nicht genau bekannt, $12240 \mathrm{~km}^{2}$ sollen Kolumbien, der Rest Venezuela gehören. Wir errechneten, gestützt auf die Karten der American Geographical Society und der Tropical Oil Company (Kolumbien) für den kolumbianischen Teil der Guajira $12000 \mathrm{~km}^{2}$, für den Venezolanischen $2050 \mathrm{~km}^{2}$. Wenn wir nur die Indianerbevölkerung berücksichtigen, kommen wir also auf eine mittlere Volksdichte von ca. 2 pro $\mathrm{km}^{2}$. Wie an anderen Orten, ergibt diese Durchschnittszahl auch hier ein etwas verzerrtes Bild, denn neben relativ dicht besiedelten Gebieten, wie dem Nordhang der Macuire um Nazaret und den relativ regenreichen Gebieten im Süden in der Nähe der Sierrra de Perijá (Gegend von Maicao) sowie um Paraguaypoa, gibt es fast menschenleere Zonen, wie das Innere der Macuire und der Jararaberge, sowie große Teile der Küstenregionen.

Die vorliegende Arbeit stützt sich auf Material, das ich im Verlaufe eines $4^{1 / 2}$ monatigen Aufenthaltes (Dez. 1949 bis Mai 1950) in der Guajirahalbinsel sammeln konnte. Außer der Südwestecke der Kolumbianischen Guajira Baja wurde dabei die ganze Halbinsel bereist und z. T. geologisch kartiert. Aus der Fülle der geologischen, geographischen und ethnologischen Beobachtungen möchte ich in diesem Artikel die Darstellung der Begräbnisbräuche der Guajira Indianer herausgreifen. Im Verlaufe der kartographischen Aufnahme der Halbinsel stießen wir immer wieder auf verlassene Friedhöfe mit sekundär bestatteten Skeletten, sodaß es uns möglich war, eine große Zahl noch gut erhaltener Schädel zu finden und zur anthropologischen Bearbeitung an Dr. E. FLEURY-CuELLO nach Caracas zu senden. Im Verlaufe der Osterwoche 1950 wurden sodann gemeinsam mit Herrn Dr. Fleury-Cuello Grabungen und Schädelmessungen in der Guajira ausgeführt und über 50 Indianer beiderlei Geschlechtes anthropometrisch vermessen. Über diese Ergebnisse der antropologischen Untersuchungen berichtet Herr Dr. Fleury-Cuello in einer eigenen Arbeit, die später erscheinen wird.

\section{BEGR Ä BN IS B R ̈̈ UCHE}

Die Guajira Indianer haben einen sehr stark entwickelten Familiensinn. Der Tod eines Guajiros bedeutet deshalb für alle Familienangehörigen einen schmerzlichen Verlust und sie bemühen sich, ihn anläßlich des Begräbnisses möglichst kräftig zum Ausdruck zu bringen. Da sich aber sehr oft nur eine kleine Zahl Familienangehöriger im Augenblick des Todes am Sterbeort befindet, muß mit dem Begräbnis zugewvartet werden, bis sich eine größere Z Zahl Leidtragender im Trauerhaus eingefunden hat. Bei dem warmen Klima der Guajira - das statistische Jahrbuch von Kolumbien gibt für Uribia ein siebenjähriges Mittel der Jahrestemperatur von $27,1^{\circ}$ an - tritt jedoch die Verwesung der Leiche sehr rasch ein, und deshalb müssen Vorkehren getroffen werden, um diese linauszuzögern. Dem (weiblichen wie männlichen) Sterbenden werden daher kurz vor dem Ableben große Mengen Rhum eingeflößt. Ist der Tote männlichen Geschlechtes, ob Säugling oder alter Mann spielt keine Rolle, werden im Moment des Todes und später beim Wegtransport der Leiche aus dem Sterbehaus Schüsse abgegeben, nach PICHON, um die bösen Geister (《guanurus») zu verscheuchen, gleichzeitig aber auch, um die To- 


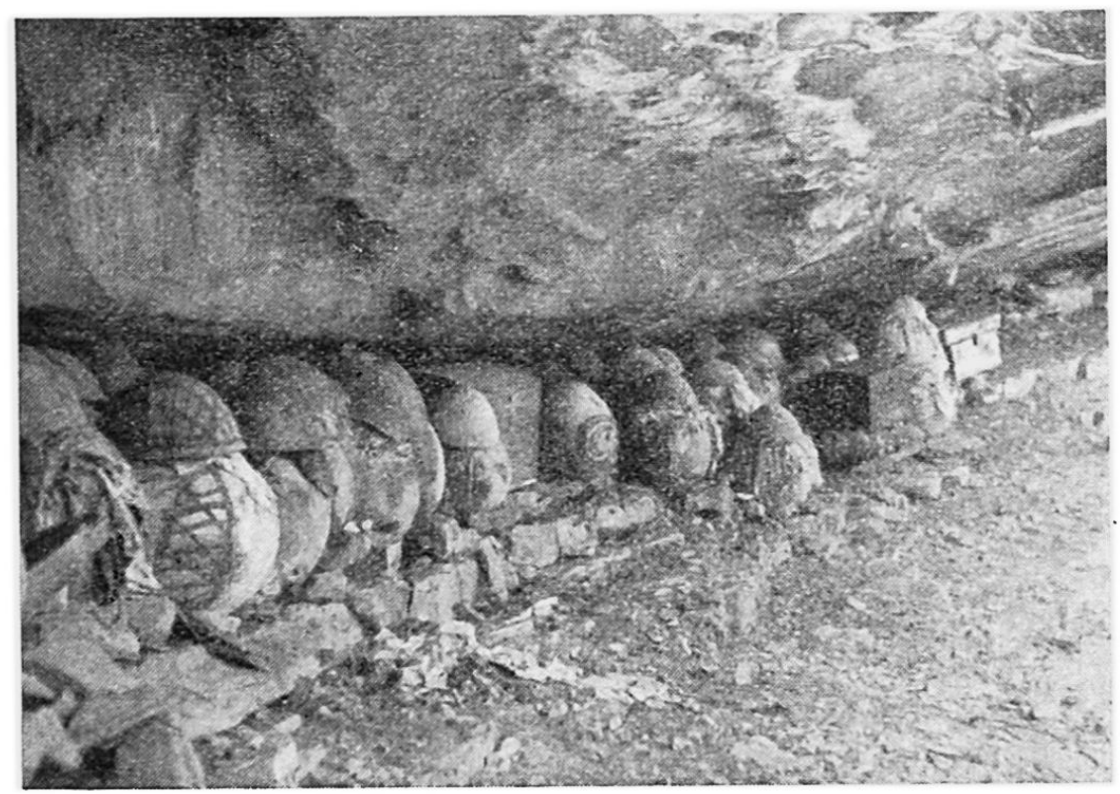

Abb. 3

Die Grabstätte Masquin im Uipanagebirge. Unter einer vorspringenden Felsbank liegen in über 100 bemalten Urnen und hölzernen Truhen die Toten der Familie Apushaima und Uriana.

Photo H. TANner, 6. 4. 1950

desnachricht bekanntzugeben. Diese wird außerdem durch Läufer und Reiter so verbreitet. Niemand konnte uns darüber Auskunft geben, warum nur beim Hinscheiden eines Mannes Schüsse abgegeben werden. Möglicherweise glaubten die Guajiros früher daran, daß die Frauen als wichtigste Stütze der Familie - es herrscht Matriarchat - besser geschützt seien gegen die Angriffe der Geister; vielleicht fäilt aber diese «Ehrensalve » deshalb ausschließlich männlichen Leichen zu, weil nur Männer mit Gewehren umgehen und die Frauen keine Beziehung zu den Waffen haben. Für die erste Hypothese spricht die Beobachtung, daß das in der Guajira übliche Wiederausgraben der 'Toten (siehe Seite 255) meist von den Frauen besorgt wird. Dem Übermittler der Todesnachricht ist es streng verboten, den $\mathrm{Na}$ men des Toten direkt zu nennen, denn vom Moment des Ablebens an darf der Tote nicht mehr beim Namen genannt werden, zum mindesten nicht in Gegenwart von Familienangehörigen des Verstorbenen. Ein Zuwiderhandeln gegen dieses Gebot konnte früher den Übeltäter der Blutrache der Leidtragenden ausliefern, im besten Fall aber zur Zahlung einer großen Entschädigung verpflichten. Heute allerdings wird (namentlich in der östlichen Küstenregion längs der Straße nach Pt. Lopez und in der Gegend um Nazaret) diesem Gebot nicht mehr durchwegs nachgelebt.

Kein Indianer konnte oder wollte uns die Ursache dieser Vorschriften erläutern : als Erklärungsversuch mögen deshalb die folgenden Beobachtungen und Erläuterungen dienen: Wie schon PicHoN andeutete, glauben die Indianer an das Vorhandensein einer Seele, die den Körper nach dem Tode verläßt. Da aber nach dem Glauben der Guajiros Körper und Seele zu Lebzeiten eine untrennbare Einheit bilden, sind sie beide zu gleichen Teilen Träger des Namens der Person. Es ist nun sehr wohl möglich, daß dic Indianer glauben, mit der Erwähnung des Namens eines Toten werde dessen Seele gezwungen, im Körper zu bleiben oder wieder in diesen zurückzukehren, wodurch den Familienangehörigen Unannehmlichkeiten entstehen könnten.

Nach dem Eintritt des Todes wird die Leiche für einige Zeit (1-2 Stunden) ruhig liegen gelassen, anscheinend, um dem Geist des Verstorbenen Gelegenheit $\mathrm{zu}$ geben, sich von Haus und Umgebung ruhig $\mathrm{zu}$ verabschieden. Dann wird der Tote gebadet und häufig, ebenfalls um die Verwesung zu verzögern, mit Salz oder Kreolin (Kreolin wird seit Jahren in der Guajira eingeführt und findet zur Wundbehandlung beim Vieh Verwendung) eingerieben. Frauen wird das Gesicht oft mit einem der 5 Farbpuder gefärbt (zwei verschiedene Rot, Schwarz, Gelb oder Braun), die die lebenden Guajiras angeblich zum Schutz gegen Sonnenbrand in dicken Lagen auf Stirn und Backen auftragen. Schließlich wird der Leichnam in ein Tuch eingepackt und in eine meist eigens für diesen $Z$ weck bestimmte Hänge- 


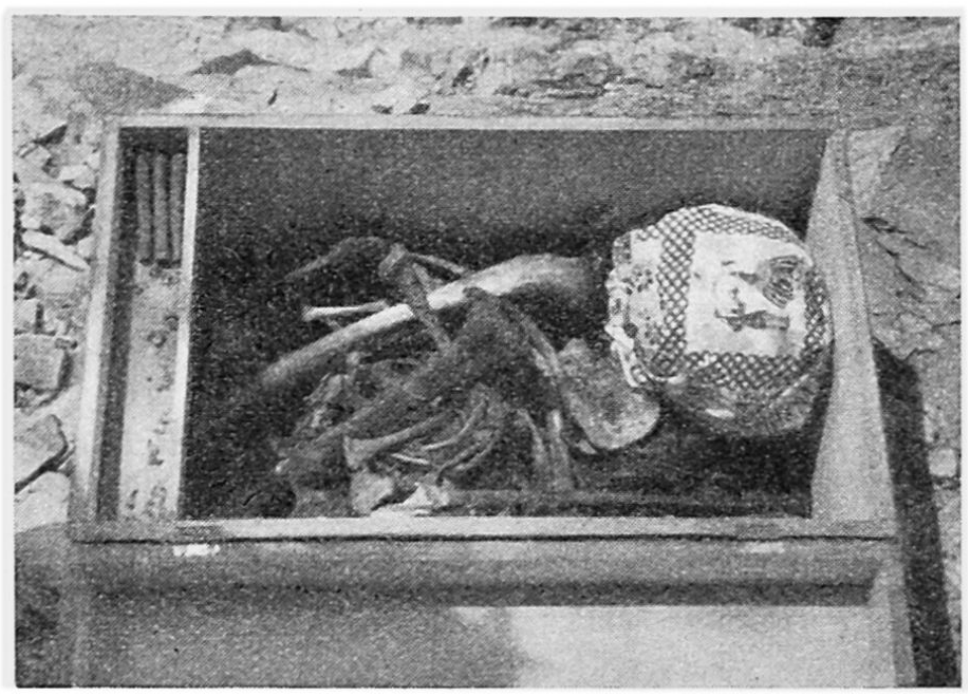

Abb. 4

Geöffnete Holztruhe aus der Grabstätte von Masquin. Rechts der in ein buntes Tuch eingepackte Schädel. Im Fach links 3 Zigarren, die dem Toten auf die Wanderschaft mitgegeben wurden.

Phot. H. Tanner, 6. 4. 1950

matte gelegt. Nach unseren Beobachtungen ruht die Leiche in ausgestreckter Lage in der Hängematte, nach STUTZER werden dem 'Toten die Knie bis zum Kinn hinauf gedrückt und dort mit Riemen festgemacht. Dann wird der Körper in Tücher und Wolldecken eingehüllt (op. cit. p. 150). Nach unseren Erkundigungen wird diese Prozedur heute nur dann vorgenommen, wenn der Tote nicht $\mathrm{zu}$ Hause stirbt und auf Lasttieren zum Ort der Trauerfeierlichkeiten transportiert werden muß. Pichon erwähnt den Fall eines toten Häuptlings, der « in der Stellung einer ägyptischen Mumie» in die Hängematte gelegt wurde (op. cit. p. 178). Bis zu diesem Zeitpunkt haben sich nur die engsten Familienangehörigen, die bisher mit dem Toten zusanmen im Hause gelebt hatten, und zwar besonders die Frauen um die Zurichtung der Leiche bemüht, während die restlichen Hausbewohner und allmählich hinzuströmende Trauergäste anscheinend unbeteiligt herumsitzen. Sobald die Leiche hergerichtet und in die Hängematte gelegt ist, stürzen sich alle Anwesenden auf einen Schlag auf die Hängematte und beginnen mit der Beweinung.

Die mit einem Tuch bedeckten Frauen kauern im allgemeinen im ersten Glied am Boden, während die Männer dahinter stehen oder knieen. Dabei pressen sie den Hut oder ein Tuch gegen das Gesicht, niemals weinen sie unbedeckten Angesichts. Oft kommt es vor, daß sich einer nach dem andern über den Toten neigt und dabei sein Gesicht möglichst in die Nähe des Leichnams bringt. Das „Weinen “ ist ein durch Mark und Bein gehendes Geheul, denn es scheint sehr wichtig zu sein, seiner Trauer durch betont lautes und vor allem durch langandauerndes Weinen Ausdruck zu geben. Nie haben wir beobachtet, daß Leidtragende Tränen vergossen hätten, dagegen sahen wir verschiedene Male Trauergäste das Schluchzen unterbrechen, sich zu den Zuschauern gesellen und mit ihnen plaudern und scherzen. Das „Weinen “ scheint bei einem Teil dieser Indianer mindestens so fern sie nicht der engern Familie angehören, zur bloßen Routine geworden zu sein, zu einer recht einträglichen Routine allerdings; denn nur wer sich am Weinen beteiligt, erhält nachher auch seinen Anteil an den Gaben, die aus dem Nachlasse des Verstorbenen an die Trauergäste verteilt werden. Je nach dessen Vermögensstand und der Bedeutung der Trauergäste erhalten diese Groß- und Kleinvieh, Fleisch, Schnaps oder Rauchwaren. Es ist den Beschenkten freigestellt, was sie mit den Tieren machen wollen. Sie können sie schlachten, zur Weiterzucht verwenden oder auch verkaufen. Früher scheint das anders gewesen zu sein, denn STUTzER erwähnt ausdrücklich, daß die Tiere nur zu Ehren des Verstorbenen verspiesen werden durften (op. cit. p. 151). Im allgemeinen erhalten alle Trauergäste ihren Anteil an der Totengabe, mit Ausnahme der engsten Familienangehörigen. Diesen ist es auch verboten, irgend etwas von den den Trauergästen verabreichten Speisen und Getränken zu genießen. Sie müssen für die Dauer der Trauerfeierlichkeiten ihre Nahrungsmittel entweder von Außenstehenden kaufen, oder sich von vor Beginn der Begräbniszeremonie beiseite gestellten Lebensmitteln ernähren.

Reicht die Hinterlassenschaft des Toten nicht aus, um allen Trauergästen Geschenke zu verabreichen, so kommt es vor, daß die näheren Verwandten aus ihrem eigenen Besitz Vieh an die Begräbnisteilnehmer abgeben. Ist dagegen nach der Verteilung der Totengeschenke nicht die ganze Hinterlassenschaft aufgebraucht, so sind die Mutter, sowie die Onkel, Neffen und Nichten der Mutterseite erbberechtigt, niemals aber die Kinder. Stirbt ein verheirateter Mann, so fallen seine sämtlichen Frauen - es herrscht Polygamie - dem ältesten Bruder zu. 


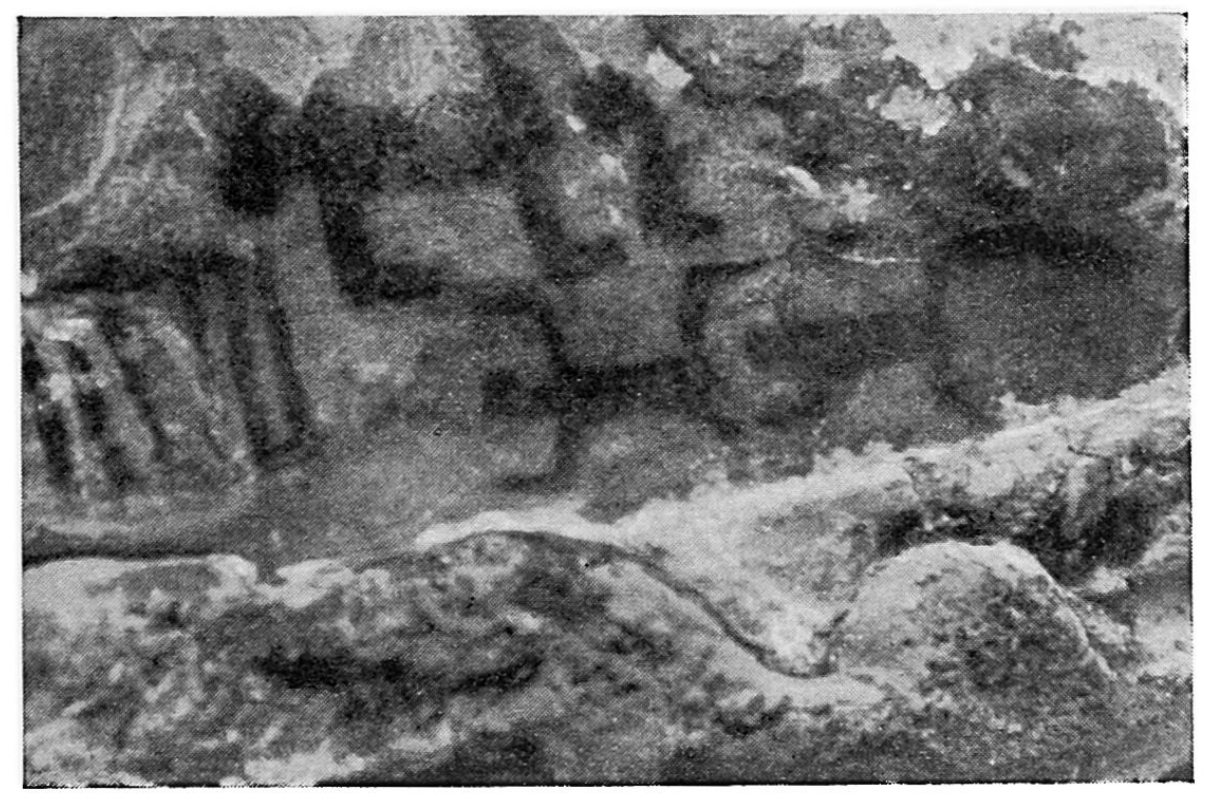

Abb. 5

Wandmalereien in

Masquin.

Photo H. TaNNer, 6. 4.1950

Der Sinn dieser Weggabe der Habe des Gestorbenen besteht nach P. JosE Augostin DE BARRANQuilla $^{4}$ (p. 184) darin. daß mit dem Tode des Besitzers auch alle seine Tiere den Tod finden sollen, damit die Geister dieser toten Tiere den Verstorbenen auf seinem zukünftigen Weg begleiten können.

Viele Indianer glauben, daß sich der Verstorbene nach dem Tode auf eine lange Wanderung begebe. Im Gebiet der Serrania de Cocinas sind wir auf die Ansicht gestoßen, daß die Indianer nach ihrem Hinscheiden auf eine Insel beim Cabo la Vela auswandern würden, wo ihnen alle Tiere aus dem Bestand ihrer Herden, die anläßlich des Begräbnisses verschenkt oder geschlachtet wurden, sowie diejenigen, die während der Lebzeit des Besitzers an Krankheiten oder Naturkatastrophen eingegangen waren, wieder zur Vertügung stünden. In der Serrania de Jarara und in der Cabo la Vela Gegend vertraten die Indianer dagegen die Ansicht, daß sich die Toten nachts in der Nähe der Grabstätten herumtreiben und daß es deshalb gefährlich sei, bei Dunkelheit in die Nähe der Gräber zu gehen. So wurden wir z. B. in der Gegend von Cashimirú (Jarara) von Indianern ernsthaft gewarnt, als wir unser Zelt am Fuße eines mit einem Grab gekrönten Hügels aufschlugen.

Bei armen Familien wird die Leiche oft schon einige Stunden nach dem Tode, oder doch am drauffolgenden Tag begraben. Bei Reichen jedoch dauern die Beweinungen oft viele Tage, ja STutzer erwähnt einen Fall, wo mit der Beerdigung mehrere Wochen zugewartet wurde. Um den Verwesungsgeruch abzuhalten, werden deshalb nach Stutzer und P. Jose Augustin die Leichen häufig nach einiger Zeit in frische Kuhhäute eingehüllt. Zum eigentlichen Begräbnis wird der Tote, hie und da mit samt der Hängematte, in einen Sarg gelegt. Dieser besteht im Normalfall aus einem ausgehöhlten Baumstamm, neuerdings werden auch aus Brettern gezimmerte Särge verwendet. Meist besitzt die Familie ihren traditionellen, abseits gelegenen Begräbnisplatz, es kommt aber auch vor, daß der Tote in oder bei der Hütte selbst oder doch in ihrer unmittelbaren Nachbarschaft begraben wird. Stutzer erwähnt das Begraben in der Hütte als Regel; wir haben derartige Gräber als Ausnahmeerscheinung in der Serranía de Cocinas beobachtet. Früher wurden die 'Toten allgemein in untiefen Gräbern beigesetzt, heute aber hat sich, außer in abgelegenen Gegenden, bei den reichen Indianern der Brauch eingebürgert, die Toten nicht mehr in der Erde, sondern in gemauerten oberirdischen Grabmälern beizusetzen.

4 P. Jose Augustin de Barranquilla. Así es la Guajira. Barranquilla, 1946. 


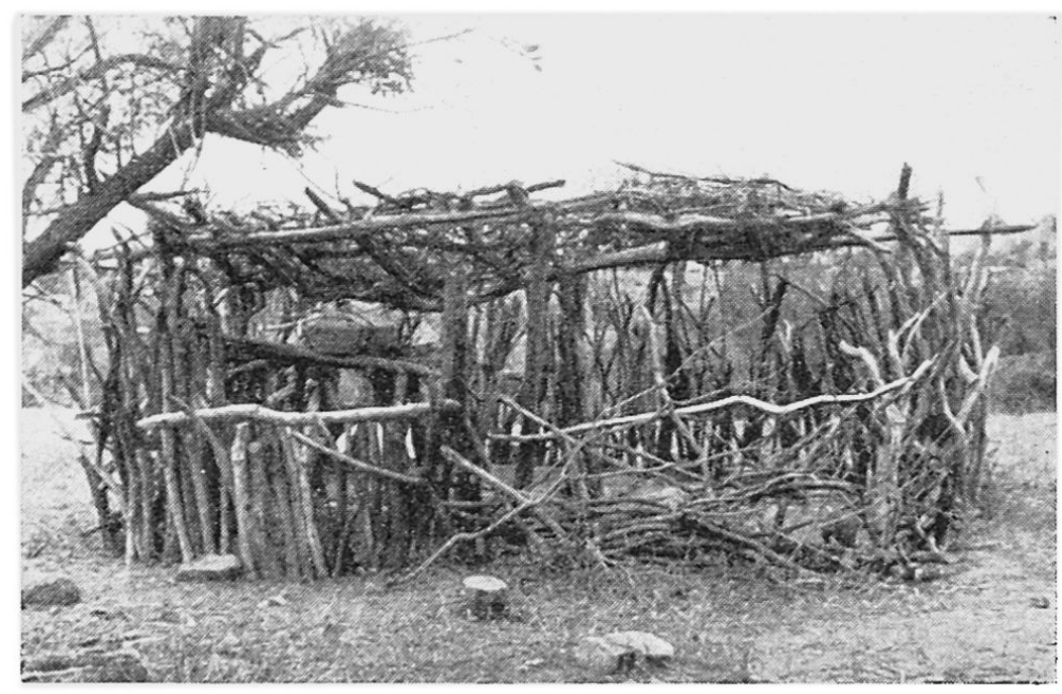

Abb. 6

Mit einem Knüppelverhau gesicherte Grabstätte zwischen Cerro Guazozop und der Serra de Cocinas.

Phot. H. TANNer, 6. 4. 1950

Diese Grabstätten sind Nachahmungen der sogenannten bovedas, die oft auf christlichen Friedhöfen Venezuelas und Kolumbiens zu finden sind. Es sind etwa mannshohe ein- oder mehrgiebelige häuserartige Bauwerke, in denen in zugemauerten Nischen die Toten beigesetzt sind. Fast alle bovedas, die wir in der Guajira beobachteten, hatten ihren Frontgiebel mit einem Kreuz verziert. Das bedeutet aber nicht, daß die Indianer, die im Grabmal beigesetzt waren, Christen gewesen wären. Das Kreuz ziert lediglich deshalb die boveda, weil auch die Gräber in christlichen Friedhöfen mit Kreuzen versehen sind. Diese Grabmäler der Indianer sind immer auf Hügeln gebaut und blendend weiß getïncht. Arme Indianer werden auch heute noch in Erdgräbern beigesetzt. Über das Grab werden Säulenkakteen gelegt, später wird oft aus Prügeln ein überdachtes luftiges Gestell rund um das Grab errichtet. Die schon früher erwähnten Grabbeigaben werden entweder in den Sarg hinein gelegt, in die boveda-Nische gestellt, oder neben das Grab auf dem Boden deponiert.

Alle Gräber mit primärbestatteten 'Toten, die wir besuchten, gleichgültig ob Erdgräber oder bovedas, waren Ost-West orientiert, und der Tote war mit dem Schädel gegen Sonnenaufgang ins Grab gelegt worden. Dies soll nach Ansicht der Indianer deshalb erfolgen, weil sonst der 'Tote « verloren» ginge, d. h. weil der Geist des Toten den Weg aus dem Grab nicht finden könnte.

Es kommt vor, daß bei der Beisetzung der Leiche, am Grab nochmals eine Beweinung stattfindet, sonst werden aber keine weiteren Zeremonien am Beisetzungsplatz abgewickelt. Die Guajiros kennen keine Priester - lediglich $\mathrm{Me}$ dizinmänner oder -frauen (butajai, butajaica $=\operatorname{der}$ Heilende). Sie spielen aber bei der gesamten Begräbniszeremonie keine besondere Rolle, höchstens daß sie als gleichberechtigte Trauergäste mitweinen. Im allgemeinen ist jedermann berechtigt, an einem Begräbnis teilzunehmen und wenn er mitgeweint hat, seinen Anteil an den Totengaben in Empfang zu nehmen. P. Jose Agostin (p. 184) erwähnt allerdings, daß in gewissen Gegenden im Ausnahmefall nur Eingeladene der Bestattung beiwohnen dürfen.

Nach der Beisetzung der Leiche kehren die Trauergäste ins Sterbehaus zurück. Wenn sich alle versammelt haben, findet nochmals eine Beweinung statt, die je nach der Bedeutung des Toten und der Ausdauer der Trauernden eine Viertelstunde bis einige Stunden dauern kann. Auch am darauffolgenden Morgen, und später noch während einiger Wochen weinen die Familienangehörigen im Hause selbst, um des Toten zu gedenken.

Am Abend des Begräbnistages und während mehrerer Wochen werden auf dem Grabe Fackeln angezündet, angeblich um dem Toten, der sich auf die Wanderung nach Cabo la Vela begibt, den Weg zu weisen. Andere Indianer versicherten uns jedoch, daß durch dieses Lichtermachen der Tote daran gehindert werden sollte, ins $\mathrm{H}$ aus zurückzukehren, um sich dort selbst ein Licht zu holen.

Zu spät kommende Trauergäste werden von einem Glied der engeren Familie zum Grab begleitet, wo sie die Beweinung nachholen. Wenn der Tote eine ange- 
sehene Person war oder sonst besonders geehrt werden soll, schlafen sie eine Nacht auf der Begräbnisstätte. Deshalb findet man in der Nähe vieler bovedas Schutzdächer und eingerammte Pfähle, damit die Leidtragenden ihre Hängematten daran befestigen können. Nach Beendigung der Trauerzeremonie erhalten auch diese $\mathrm{Zu}$ spätgekommenen noch ihre Totengaben.

Bei der ersten Begegnung mit einem engeren Familienglied des Verstorbenen, das aus irgend einem Grund nicht am Begräbnis teilnehmen konnte, drücken die Guajiros ihm ihr Beileid dadurch aus, daß sie sich gegenüber dem Leidtragenden auf den Boden kauern und ihn längere Zeit möglichst laut anschluchzen.

Für Ėrmordete wird nur eine kurze Begräbnisfeier veranstaltet, an der gewöhnlich nur Frauen teilnehmen. Würde man eine vollständige Trauerfeier mit langer Beweinung, Totengaben usw. durchführen, so wäre nach dem Glauben der Indianer keine wirksame Blutrache mehr möglich. Wir begegneten auch der Ansicht, daß Männer, die sich an einer derartigen Trauerfreier beteiligten, sich in Feiglinge verwandeln würden. Die 'Trauerfeier wird nachgeholt, wenn der Mord gerächt ist, sei es durch Tötung des Mörders oder durch Bezahlung einer Entschädigungssumme, die von der Familie des Mörders entrichtet werden muß.

Früher wurde die Hütte, in der der Tod erfolgte, immer abgerissen oder ihr Standort verlegt. So beobachtete StuTZER, wie am Nordwestende der Macuire eines Morgens die Indianer mit dem Dach einer Hütte über die Steppe eilten, um es einige Kilometer entfernt wieder aufzubauen. Auch heute noch wird das Sterbehaus gewöhnlich verlassen, hie und $\mathrm{da}$ auch angezündet, aber uns wurde von verschiedenen Fällen berichtet, wo dieser Regel nicht mehr nachgelebt wurde.

Mit der Beisetzung der Toten in Grab oder bovedas haben diese jedoch noch nicht ihre endgültige Ruhestätte gefunden, denn die Guajiros kennen wie auch die anderen Aruakstämme Venezuelas, eine zweimalige Bestattung ihrer Verstorbenen. Nach einigen Jahren (meistens 2-3 Jahren) wird eine neuerliche Trauerfeierlichkeit veranstaltet, der allerdings gewöhnlich nur noch die engeren Familienangehörigen und eingeladene Trauergäste beiwohnen. Gemeinsam zieht die Trauergesellschaft aufs Grab. Nach dessen Öffnung werden die Knochen meist durch eine alte, weibliche Verwandte von Hand aus dem Sarg geholt, von allfällig noch anhaftenden Fleischresten gereinigt und in einen irdenen Krug gelegt. Der Schädel wird gewöhnlich in ein Tuch eingewickelt und nachher den Knochen beigefügt. Die Urne wird mit einem 'Tuch verschlossen und zugebunden, als besonderer Schutz wird noch ein irdener Deckel über die Öffnung gestülpt. Wenn die Knochen im Kruge verpackt sind, beginnt eine erste Beweinung über der Urne, eine weitere erfolgt abends und während der Nacht zu Hause. Anläßlich der endgültigen Beisetzung am folgenden Tag findet, sei es in einer boveda - die aber nicht mehr Ost-West orientiert zu sein braucht - oder in der Nähe der bisherigen Begräbnisstätte im Boden, in aiggelegenen Dünenregionen, in Felsspalten oder unter vorspringenden Felsen, schließßlich eine dritte und letzte Beweinung statt.

Wir haben viele derartige Begräbnisplätze gefunden, darunter im Uipanagebirge einen eigentlichen Friedhof. Unter einer vorspringenden Felsbank liegt dort am Berghang an schwer zugänglicher Stelle eine Ansammlung von 100 bemalten Urnen und 7 hölzernen Truhen. Eigentümlicherweise liegen in einzelnen Urnen nicht nur ein, sondern mehrere Skelette bestattet. Die Urnen sind auf hellbraunrotem Grund mit dunkelbraunen Ornamenten bemalt, die sich nicht von Ornamenten unterscheiden, die die Wasserkrüge der Guajiros schmücken.

Die Holztruhen beherbergen möglicherweise Leichen von Indianern, die nicht in der Guajira, sondern in der Fremde gestorben, später exhumiert und in der Truhe in die Heimat transportiert wurden. Es scheint sehr oft vorzukommen, daß die Angehörigen von in Maracaibo verstorbenen Indianern die Leiche aus den Friedhöfen stehlen und in die Guajira transportieren. Wir haben verschiedene derartige Transporte beobachtet. Die Felsdecke über den Urnen ist mit Zeichnungen verziert. Neben dem in 
weißer Farbe ausgeführten Stammeszeichen der Familien Uriana und Apushaina fanden wir aber auch eigenartige in Reihen angeordnete etwa $10 \mathrm{~cm}$ große Menschenfiguren in rotbrauner Farbe. Nach dem Erhaltungszustand zu schließen, scheinen diese Malereien ein beträchtliches Alter zu haben, was dadurch bestätigt wird, daß kein Indianer in der Gegend uns über die Herkunft oder Bedeutung dieser Figuren Auskunft geben konnte. Möglicherweise stellen die Figurenketten, die mit ausgestreckten Gliedern daliegende Menschen darstellen, wobei der Kopf des Hintermannes zwischen den Beinen des Vordermannes ruht, die Aufeinanderfolge der hier bestatteten Generationen dar. Bei der Untersuchung der Graburnen sind wir oft auf rotgefärbte Schädel und Knochen gestoßen. Es scheint, daß das Färben der Schädel (mit einem rotbraunen Pulver) früher verbreitet war. Heute aber wird es kaum mehr geübt, denn keiner der Bewohner der Guajira die wir befragten, ob Indianer, Mestizen oder Missionare, hatten je derartigen Bemalungen von Schädeln beigewohnt. Bei den andern Aruakstämmen Venezuelas, wie auch bei andern Indianern, z. B. den Motilones ist dagegen die Schädelbemalung noch heute üblich.

Auch bei der zweiten Bestattung erhält der Tote Grabbeigaben. Wir haben besonders viele Rhumflaschen, dann aber auch Zigarren, Totumas mit Lebensmitteln und Behälter mit Schmuck gefunden. Ebenso werden an die Trauergäste wieder Geschenke abgegeben, diesmal jedoch nicht mehr lebende Tiere, sondern nur noch Fleisch und Getränke.

$\mathrm{Da}$ nach der Ansicht der Guajiros durch das Ausgraben der Knochen der Geist des Toten gestört wird, muß die Person, die das Ausgraben besorgt hat, vor Angriffen der Geister geschützt werden. Während der ersten Nacht - nach P. Jose Augustin während der ersten zwei Nächte - darf sie nicht schlafen. Sie legt sich in eine zu kleine Hängematte, man singt an ihrer Seite die ganze Nacht und gießt ihr Wasser ins Gesicht, falls sie trotzdem einschlafen sollte. Interessanterweise werden diese Vorsichtsmaßnahmen aber nur bei der ersten Ausgrabung durchgeführt. Übersteht die Ausgrabende diese erste kritische Nacht heil, so ist sie gegen die Angriffe der Geister gefeit und muß bei späteren Ausgrabungen nicht mehr besonders geschützt werden. Nach jeder Ausgrabung ist es ihr jedoch streng verboten, mit ihren eigenen $\mathrm{Händen} \mathrm{zu} \mathrm{essen} \mathrm{oder} \mathrm{jemanden} \mathrm{(auch} \mathrm{sich} \mathrm{selber)} \mathrm{zu}$ berühren. Die Nahrung wird ihr oft während mehreren Wochen eingeflößt und damit sie sich selber kratzen kann, werden ihr auf der Innenseite der Handgelenke Stäbchen befestigt. Nach P. Jose Aucustin (op. cit. p. 190) muß die Totengräberin während dieser Zeit im 'Essen $\mathrm{Maß}$ halten, ja, sie soll sogar von Zeit zu Zeit fasten. Während dieser Zeit wird sie auch meist von Fremden abgesondert und verläßt ihre Hütte nicht.

\section{OSSERVAZIONI ETNOLOGICHE SULLA PENISOLA DI GUAJIRA}

La vita economica dei 25000 indiani che vivono nella Penisola di Guajira è sottoposta a una brève analisi. La parte principale del lavoro è invece riservata alla descrizione dei riti di sepoltura che consistono in un ripetersi dell'atto del seppellimento e delle corrispondenti cerimonie.

OBSERVATIONS ETHNOLOGIQUES SUR LA PRESQU'TिLE DE LA GOAJIRA

La presqu'île de la Goajira est restée un damaine des Indiens Goajiros auxquels cette étude est consacrée. Sa partie principale s'applique à la description de leurs funérailles, qui se caractérisent par des enterrements répetés avec des cérémonies spécifiques.

\section{GEOGRAPHIE UND KARTOGRAPHIE AN DER JAHRESVERSAMMLUNG DER SCHWEIZERISCHEN NATURFORSCHENDEN GESELLSCHAFT I95I}

\section{ERICH Schwabe}

Die Tagung der Schweiz. Naturforschenden Gesellschaft fand dieses Jahr unter zahlreicher Beteiligung vom 29. September bis 1. Oktober in Luzern statt. Die Leuchtenstadt empfing ihre Gäste nicht gerade mit strahlendem Himmel, doch war 